\title{
18. THE SEDIMENTARY DEPOSITS OF SUIKO SEAMOUNT (LEG 55, SITE 433): FROM THE REEF ENVIRONMENT TO THE PELAGIC SEDIMENTATION
}

\author{
Anne Marie Karpoff, Institut de Géologie, Strasbourg, France
}

\section{INTRODUCTION}

The four holes (including a re-entry hole) drilled at Site 433 allow determination of the sedimentary sequence of Suiko Seamount in the Emperor chain. The holes are in a small graben basin situated within a lateral lagoon on the seamount. The sedimentary deposits range from the Paleocene to the upper Pliocene and are not uniform and continuous. A major hiatus exists at the top of the lower Eocene reef sediment, below the lower and upper Miocene pelagic sediments. The depositional history and succession of environments are shown by mineralogical and geochemical changes in the sediments.

\section{LITHOLOGY}

The diversified sedimentary sequences recovered from the four holes $(433,433 \mathrm{~A}, 433 \mathrm{~B}$, and $433 \mathrm{C})$ are described in the site reports (this volume), and the biostratigraphic results are summarized by Koizumi, Butt, Ling, and Takayama (this volume). The composite sequence comprises six lithologic units the thicknesses of which can only be approximated from the recovered material because of the drilling disturbance. These lithologic units are as follows:

Unit 1: $0.0-1.5$ meters: Upper Pliocene and lower Pleistocene foraminiferal nannofossil ooze.

Unit 2: 1.5-14.5 meters: Upper Pliocene sandy diatom-nannofossil ooze.

14.5-43.0 meters: Upper Miocene and lower Pliocene marly siliceous nannofossil ooze.

Unit 3: 43.0-51.5 meters: Upper Miocene calcareous ooze and chalk.

Unit 4: 51.5-52.0 meters: Lower Miocene siliceous ooze.

52.0-52.5 meters: Lower Miocene sandy tuffaceous mud.

Unit 5: 52.5-168.0 meters: Late and middle Paleocene reef sand, sandy mud with algal concretion, and calcarenite.

Unit 6: 177.5-181.5 meters: Volcanic reef sand interlayered between basalt Flow Units 1 and 2.

In the upper part of the sedimentary sequence, the lithologic units do not correspond exactly to the paleontological partitions. There are several biostratigraphic hiatuses: between Units 1 and 2; in Unit 2; and the ma- jor hiatus between Units 3 and 4, in which can be differentiated two separate periods of hiatus - at the lower part of Unit 3 during middle Miocene, and before the reef sedimentation (Unit 5) in the Paleocene. From Unit 4 to Unit 1 the fossil assemblages are generally reworked, and the sediment accumulation rate of this section, about $13 \mathrm{~m} / \mathrm{m} . \mathrm{y}$., is relatively high.

\section{MINERALOGY}

The mineralogical analyses using the X-ray diffraction techniques as described by Karpoff et al. (this volume) were performed on bulk sediments. Descriptive data are given in Table 1. The uniform calcareous character of the deposits masks, on the X-ray diffraction charts, the occurrence of small amounts of other minerals.

Calcite prevails and forms the pelagic biogenic component as foraminifers and nannofossils in Units 1 to 4 and the reef deposits in the lower Units 5 and 6.

Amorphous silica, from the siliceous biogenic component as diatoms, is well represented in Unit 2.

Clays include illite, kaolinite, smectites, and trace amounts of chlorite; they are abundant in Units 2, 3, and 4.

Detrital silicates, as volcanic glass, quartz, plagioclases, and trace amounts of amphiboles, occur in Units 1 and 2 , and prevail in the coarse fraction $(>63 \mu \mathrm{m})$ of the sandy volcanic layer (Unit 4).

Authigenic minerals occur in some units. Unit 2 contains small amounts of carbonate-apatite; this mineral occurs also in Unit 4 associated with barite and the zeolite harmotome. In the reef sand (Unit 5), gypsum appears in trace amounts in some samples, and pyrite forms concretions which can be separated from the bulk material (e.g., Sample 433A-8-1, 180-182 cm).

The mineral assemblage - calcite, clays, amorphous silica, and detrital components - is constant in the upper part of the pelagic deposits from Unit 1 to Unit 3, with only a change in the proportions of minerals. The sandy volcanic mud is mineralogically distinct from the pelagic sediments and the calcareous lagoonal formation containing low concentrations of gypsum, pyrite, and clays. The occurrence of authigenic zeolites in this section, described by McKenzie et al. (this volume), is not observable on the X-ray diffraction charts.

\section{GEOCHEMISTRY}

\section{Results and Characteristics of the Successive Lithologic Units}

The bulk chemical compositions of the samples from Site 433 are given in Tables 2 and 3. The same bulk ma- 
TABLE 1

Mineralogical Results from X-Ray Diffraction of Non-Oriented Powders for Site 433 (Holes 433, 433A, 433B, 433C). Descriptive Data Only

\begin{tabular}{|c|c|c|c|c|c|c|c|c|c|}
\hline $\begin{array}{c}\text { Sample } \\
\text { (Interval in } \mathrm{cm} \text { ) }\end{array}$ & Unit & $\frac{\frac{\pi}{0}}{\frac{\pi}{\delta}}$ & 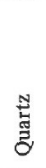 & 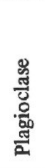 & 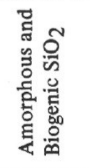 & 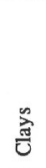 & 营 & 莺 & Other Minerals \\
\hline $\begin{array}{l}433-1-1,60-62 \\
\quad-1-1,129-131\end{array}$ & $\begin{array}{l}1 \\
1\end{array}$ & $\begin{array}{l}+ \\
+\end{array}$ & $\begin{array}{l}+ \\
+\end{array}$ & $\begin{array}{l}+ \\
+\end{array}$ & & + & + & & Tr Amphibole \\
\hline $\begin{array}{r}433 \mathrm{~A}-1-1,7-9 \\
-2-1,70-72 \\
-3-1,80-85 \\
-3-6,21-23\end{array}$ & $\begin{array}{l}1 \\
1 \\
1 \\
1\end{array}$ & $\begin{array}{l}+ \\
+ \\
+ \\
+\end{array}$ & $\begin{array}{l}+ \\
+\end{array}$ & $\begin{array}{l}+( \\
+\end{array}$ & dd K-feld) & & $\begin{array}{l}+ \\
+\end{array}$ & & \\
\hline $\begin{array}{c}433-1-2,40-42 \\
-1-2,127-129 \\
-1-3,7-9 \\
-1-3,104-106 \\
-1-4,26-28\end{array}$ & $\begin{array}{l}2 \\
2 \\
2 \\
2 \\
2\end{array}$ & $\begin{array}{l}+ \\
+ \\
+ \\
+ \\
+\end{array}$ & $\begin{array}{l}+ \\
\text { Tr } \\
+ \\
+\end{array}$ & $\begin{array}{l}+ \\
+ \\
+ \\
+\end{array}$ & + & + & + & & Amphibole? \\
\hline $\begin{array}{c}433 A-4-1,7-8 \\
-4-2,66-68 \\
-4-2,68-70 \\
-4-2,100-105 \\
-4-3,55-60 \\
-4-3,85-90 \\
-4-4,60-62 \\
-4-6,92-95 \\
-5-2,130-132 \\
-5-4,20-22 \\
5-5,110-112\end{array}$ & $\begin{array}{l}2 \\
2 \\
2 \\
2 \\
2 \\
2 \\
2 \\
2 \\
2 \\
2 \\
2\end{array}$ & $\begin{array}{l}+ \\
+ \\
+ \\
+ \\
+ \\
+ \\
+ \\
+ \\
+ \\
+ \\
+\end{array}$ & $\begin{array}{l}+ \\
+ \\
+ \\
+ \\
+ \\
+ \\
+ \\
+ \\
+ \\
+\end{array}$ & $\begin{array}{l}\operatorname{Tr} \\
\operatorname{Tr}\end{array}$ & $\begin{array}{l}+ \\
+ \\
+ \\
+ \\
+ \\
+ \\
+\end{array}$ & $\begin{array}{l}+ \\
+ \\
+ \\
+ \\
+ \\
+ \\
+ \\
+ \\
+\end{array}$ & $\begin{array}{l}+ \\
+ \\
+ \\
+ \\
\text { Tr } \\
+ \\
+\end{array}$ & $\begin{array}{l}\text { Tr } \\
\text { Tr }\end{array}$ & \\
\hline $\begin{array}{l}-6-1,50-53 \\
-6-3,140-144\end{array}$ & $\begin{array}{l}3 \\
3\end{array}$ & + & $\operatorname{Tr}$ & & & & $\begin{array}{l}\operatorname{Tr} \\
+\end{array}$ & & \\
\hline $\begin{array}{l}-6-6,60-62 \\
-6-6,120-122\end{array}$ & $\begin{array}{l}3 \\
3\end{array}$ & + & $\stackrel{+}{T_{r}}$ & & & + & $\begin{array}{c}+ \\
\mathrm{Tr}\end{array}$ & & \\
\hline $\begin{array}{l}-6-7,20-22 \\
-6-7,36-38\end{array}$ & $\begin{array}{l}4 \\
4\end{array}$ & $\begin{array}{l}+ \\
+\end{array}$ & + & + & $\begin{array}{l}+ \\
+\end{array}$ & $\begin{array}{l}+ \\
+\end{array}$ & & + & Barite \\
\hline$-6, \mathrm{CC}$ & 4 & + & + & + & & + & & + & $\begin{array}{l}\text { Barite-zeolites } \\
\text { (harmotome) }\end{array}$ \\
\hline $\begin{array}{l}-7-1,90-92 \\
-7-6,100-102 \\
-8-1,120-122 \\
-8-1,120-122 b \\
-8-3,15-17\end{array}$ & $\begin{array}{l}5 \\
5 \\
5 \\
5 \\
5\end{array}$ & $\begin{array}{l}+ \\
+ \\
+ \\
+\end{array}$ & & & & + & & & Pyrite \\
\hline $\begin{array}{l}-8-7,40-42 \\
-9-2,30-32 \\
-10-1,71-73 \\
-10-7,3-5 \\
-12-1,72-74 \\
-13-1,20-22 \\
-16-1,58-60 \\
-16, C C(4-7) \\
-19-1,23-25\end{array}$ & $\begin{array}{l}5 \\
5 \\
5 \\
5 \\
5 \\
5 \\
5 \\
5 \\
5\end{array}$ & $\begin{array}{l}+ \\
+ \\
+ \\
+ \\
+ \\
+ \\
+ \\
+ \\
+\end{array}$ & & & & & + & & Gypsum \\
\hline $\begin{array}{c}\text { 433B-1-4, } 119-120 \\
-1-4,120-126 \\
-2-1,0-150 \\
-3-1,20-60\end{array}$ & $\begin{array}{l}5 \\
5 \\
5 \\
5\end{array}$ & $\begin{array}{l}+ \\
+ \\
+ \\
+\end{array}$ & & & & & + & & \\
\hline $433 \mathrm{C}-3-2,103-106$ & 6 & + & & + & + & & & & \\
\hline
\end{tabular}

terial as studied in the X-ray diffraction analyses were analyzed here, using the spectrometry techniques described by Karpoff et al. (this volume). Results are shown in Figure 1.

In accordance with the mineralogical composition, the two prevalent elements are calcium and silicon, in proportions which vary with the lithology. The variation of the $\mathrm{Si} / \mathrm{A} 1$ ratio (Table 4) follows the relative abundances of silicates as clays and volcanic fragments and of amorphous biogenic silica as diatoms.

Unit 1. The deposits are composed of calcareous material (high $\mathrm{Ca}, \mathrm{Sr}$ ) associated with clays and other detrital minerals, and are characterized by a low $\mathrm{Si} / \mathrm{A} 1$ ratio; they contain relatively high concentrations of $\mathrm{Fe}$, $\mathrm{Ti}, \mathrm{Mg}, \mathrm{Na}, \mathrm{K}$, and the trace elements $\mathrm{Mn}, \mathrm{V}, \mathrm{Ni}, \mathrm{Cr}$, $\mathrm{Cu}, \mathrm{Zn}$, and $\mathrm{Pb}$.
Unit 2. From top to bottom, the silicon content decreases with a correlative increasing calcium content. The greater amount of diatoms and other siliceous organisms in the upper part of this unit is indicated by the increase in the $\mathrm{Si} / \mathrm{Al}$ ratio and in silicon, barium, and lead contents. The occurrence of $\mathrm{Ba}$ and $\mathrm{Pb}$ in the siliceous organisms is well known, and high concentrations of these elements in pelagic siliceous oozes are often described, (El Wakeel and Riley, 1961; Arrhenius, 1963, Brongersma-Sanders, 1967; Turekian, 1968; Chow and Goldberg, 1970; Boström et al., 1973; Hoffert et al., 1978).

The authigenic silicates in the same upper part of Unit 2 are clays, and their occurrence is marked by an increased boron content (Landergreen and Carjaval, 1969; Kukal, 1971). In this unit, trace elements such as $\mathrm{Co}, \mathrm{Cr}, \mathrm{Ni}, \mathrm{Zn}, \mathrm{Cu}$, and $\mathrm{Mn}$ increase with increasing $\mathrm{Fe}$, $\mathrm{Mg}$, $\mathrm{Na}$, and $\mathrm{K}$.

Unit 3. This lithologic unit has the same composition as the overlying part of Unit 2, with a slight increase in the silicon content.

Unit 4. The two sub-units, siliceous ooze and volcanic sandy mud, have the lowest carbonate contents and correspondingly higher concentrations of other elements. The two sub-units differ in their silica and boron contents on the one hand, and on the other in their iron and trace-element contents, corresponding to the siliceous organisms and authigenic clays, and the volcanic detrital fragments, respectively. The presence of other minerals, such as barite and carbonate-apatite, is indicated in the two levels by the barium and phosphorus contents. These two layers are of the same geochemical facies, and differ only in the planktonic and mineral contributions.

Units 5 and 6. The reef calcareous sandy mud is characterized by its low contents of all elements other than calcium. A peculiarity of these calcareous deposits is their very low strontium contents. The pyrite concretion contains small amounts of silicon, barium, and nickel. Unit 6 is a mixture of lagoonal sand and volcanic fragments, and accordingly contains higher concentrations of elements from the volcanic phases.

\section{Strontium Variations in the Sedimentary Deposits of Site $\mathbf{4 3 3}$}

The positive correlation between strontium and calcium in carbonate sediments is well known. At Site 433, the calcareous biogenic phases differ, below and above the sandy volcanic mud (Unit 4), in their strontium contents. According to data from Turekian (1964), the $\mathrm{Sr} / \mathrm{Ca}$ ratios range from 20 to 5 in reef formations and deep-sea carbonates, respectively. In the Site 433 lagoonal sandy mud (Units 5 and 6), the $\mathrm{Sr} / \mathrm{Ca}$ ratio is very low, and increases in the overlying volcanic layer and diatom-calcareous oozes (Table 4). The $\mathrm{CaO}-\mathrm{Sr}$ diagram (Figure 2) shows the relative positions of the different lithologic units. In the pelagic siliceous and calcareous oozes (Units 1,2, and 3), the $\mathrm{Sr}$ and $\mathrm{CaO}$ contents are strongly correlated, with normal concentrations. In the volcanic layer and the siliceous ooze from Unit 4 with a low biogenic calcium content, the Sr con- 
TABLE 2

Chemical Analyses of Sedimentary Deposits from Site 433, Leg 55, Suiko Seamount. Major Elements (\%)

\begin{tabular}{|c|c|c|c|c|c|c|c|c|c|c|c|c|c|}
\hline $\begin{array}{c}\text { Sample } \\
\text { (Interval in } \mathrm{cm} \text { ) }\end{array}$ & Unit & $\mathrm{SiO}_{2}$ & $\mathrm{Al}_{2} \mathrm{O}_{3}$ & $\mathrm{MgO}$ & $\mathrm{CaO}$ & $\mathrm{Fe}_{2} \mathrm{O}_{3}$ & $\mathrm{Mn}_{3} \mathrm{O}_{4}$ & $\mathrm{TiO}_{2}$ & $\mathrm{BaO}$ & $\mathrm{Na}_{2} \mathrm{O}$ & $\mathrm{K}_{2} \mathrm{O}$ & Ign & Total \\
\hline $\begin{array}{l}433-1-1,60-62 \\
\quad-1-1,129-131\end{array}$ & $\begin{array}{l}1 \\
1\end{array}$ & $\begin{array}{l}35.6 \\
36.3\end{array}$ & $\begin{array}{l}7.8 \\
7.6\end{array}$ & $\begin{array}{l}1.35 \\
1.51\end{array}$ & $\begin{array}{l}27.5 \\
26.1\end{array}$ & $\begin{array}{l}3.0 \\
3.4\end{array}$ & $\begin{array}{l}0.057 \\
0.047\end{array}$ & $\begin{array}{l}0.36 \\
0.35\end{array}$ & $\begin{array}{l}0.09 \\
0.15\end{array}$ & $\begin{array}{l}1.35 \\
1.13\end{array}$ & $\begin{array}{l}0.62 \\
0.69\end{array}$ & $\begin{array}{l}23.12 \\
22.74\end{array}$ & $\begin{array}{l}100.85 \\
100.02\end{array}$ \\
\hline $\begin{array}{r}433 \mathrm{~A}-1-1,7-9 \\
-2-1,70-72 \\
-3-1,80-85 \\
-3-3,21-23\end{array}$ & $\begin{array}{l}1 \\
1 \\
1 \\
1\end{array}$ & $\begin{array}{r}37.3 \\
45.8 \\
12.0 \\
9.8\end{array}$ & $\begin{array}{l}7.5 \\
9.3 \\
0.5 \\
0.7\end{array}$ & $\begin{array}{l}1.54 \\
1.87 \\
0.38 \\
0.32\end{array}$ & $\begin{array}{l}24.4 \\
18.5 \\
47.0 \\
49.1\end{array}$ & $\begin{array}{l}3.7 \\
4.8 \\
0.2 \\
0.2\end{array}$ & $\begin{array}{l}0.055 \\
0.061 \\
0.012 \\
0.013\end{array}$ & $\begin{array}{l}0.38 \\
0.45 \\
0.03 \\
0.03\end{array}$ & $\begin{array}{l}0.14 \\
0.14 \\
0.09 \\
0.09\end{array}$ & $\begin{array}{l}1.31 \\
1.80 \\
0.05 \mathrm{H} \\
0.05 \mathrm{H}\end{array}$ & $\begin{array}{l}0.80 \\
1.28 \\
0.05 \mathrm{H} \\
0.05 \mathrm{H}\end{array}$ & $\begin{array}{l}21.25 \\
14.97 \\
40.06 \\
40.15\end{array}$ & $\begin{array}{r}98.57 \\
98.97 \\
100.37 \\
100.41\end{array}$ \\
\hline $\begin{array}{c}433-1-2,40-42 \\
-1-2,127-129 \\
-1-3,7-9 \\
-1-3,104-106 \\
-1-4,26-28\end{array}$ & $\begin{array}{l}2 \\
2 \\
2 \\
2 \\
2\end{array}$ & $\begin{array}{l}46.4 \\
26.8 \\
34.7 \\
28.1 \\
28.5\end{array}$ & $\begin{array}{l}7.1 \\
0.6 \\
3.1 \\
1.7 \\
0.5\end{array}$ & $\begin{array}{l}1.71 \\
0.50 \\
0.92 \\
0.74 \\
0.36\end{array}$ & $\begin{array}{l}19.9 \\
37.5 \\
31.1 \\
35.0 \\
36.4\end{array}$ & $\begin{array}{l}4.6 \\
0.4 \\
1.8 \\
1.1 \\
0.1 \mathrm{H}\end{array}$ & $\begin{array}{l}0.046 \\
0.010 \mathrm{H} \\
0.022 \\
0.018 \\
0.010 \mathrm{H}\end{array}$ & $\begin{array}{l}0.37 \\
0.04 \\
0.16 \\
0.08 \\
0.02\end{array}$ & $\begin{array}{l}0.20 \\
0.15 \\
0.16 \\
0.17 \\
0.12\end{array}$ & $\begin{array}{l}1.65 \\
0.09 \\
0.49 \\
0.26 \\
0.07\end{array}$ & $\begin{array}{l}0.87 \\
0.05 \mathrm{H} \\
0.34 \\
0.11 \\
0.05 \mathrm{H}\end{array}$ & $\begin{array}{l}17.70 \\
33.87 \\
28.12 \\
32.17 \\
33.76\end{array}$ & $\begin{array}{r}100.55 \\
100.01 \\
100.91 \\
99.45 \\
99.89\end{array}$ \\
\hline $\begin{array}{c}433 \mathrm{~A}-4-1,7-8 \\
-4-2,66-68 \\
-4-2,68-70 \\
-4-2,100-105 \\
-4-3,55-60 \\
-4-3,85-90 \\
-4-4,60-62 \\
-4-6,92-95 \\
-5-2,130-132 \\
-5-4,20-22 \\
-5-5,110-112\end{array}$ & $\begin{array}{l}2 \\
2 \\
2 \\
2 \\
2 \\
2 \\
2 \\
2 \\
2 \\
2 \\
2\end{array}$ & $\begin{array}{l}33.8 \\
58.9 \\
59.3 \\
57.2 \\
45.3 \\
36.7 \\
35.4 \\
30.3 \\
18.8 \\
19.2 \\
26.7\end{array}$ & $\begin{array}{l}1.0 \\
3.2 \\
3.4 \\
1.6 \\
1.5 \\
1.1 \\
1.2 \\
1.3 \\
0.4 \\
0.8 \\
1.3\end{array}$ & $\begin{array}{l}0.55 \\
1.73 \\
1.88 \\
1.02 \\
0.92 \\
0.59 \\
0.52 \\
0.45 \\
0.33 \\
0.52 \\
0.76\end{array}$ & $\begin{array}{l}33.2 \\
10.7 \\
10.0 \\
15.8 \\
23.5 \\
30.7 \\
30.3 \\
35.2 \\
43.8 \\
43.3 \\
37.1\end{array}$ & $\begin{array}{l}0.6 \\
3.7 \\
4.3 \\
0.9 \\
1.0 \\
0.5 \\
0.8 \\
0.5 \\
0.2 \\
0.3 \\
0.6\end{array}$ & $\begin{array}{l}0.019 \\
0.022 \\
0.026 \\
0.010 \mathrm{H} \\
0.024 \\
0.015 \\
0.022 \\
0.022 \\
0.020 \\
0.012 \\
0.014\end{array}$ & $\begin{array}{l}0.05 \\
0.17 \\
0.18 \\
0.08 \\
0.09 \\
0.05 \\
0.06 \\
0.05 \\
0.03 \\
0.04 \\
0.07\end{array}$ & $\begin{array}{l}0.27 \\
0.73 \\
0.67 \\
0.53 \\
0.40 \\
0.30 \\
0.32 \\
0.20 \\
0.12 \\
0.19 \\
0.31\end{array}$ & $\begin{array}{l}0.21 \\
1.87 \\
2.13 \\
1.08 \\
0.90 \\
0.25 \\
0.36 \\
0.22 \\
0.05 \\
0.05 \\
0.12\end{array}$ & $\begin{array}{l}0.12 \\
1.63 \\
1.85 \\
1.09 \\
0.73 \\
0.20 \\
0.25 \\
0.20 \\
0.05 \mathrm{H} \\
0.05 \mathrm{H} \\
0.05 \mathrm{H}\end{array}$ & $\begin{array}{l}30.69 \\
15.76 \\
14.63 \\
20.43 \\
25.31 \\
29.60 \\
30.31 \\
31.47 \\
36.76 \\
35.94 \\
33.61\end{array}$ & $\begin{array}{r}100.51 \\
98.41 \\
98.37 \\
99.74 \\
99.67 \\
100.01 \\
99.64 \\
99.91 \\
100.56 \\
100.40 \\
100.63\end{array}$ \\
\hline $\begin{array}{l}-6-1,50-53 \\
-6-3,140-144 \\
-6-6,60-62 \\
-6-6,120-122\end{array}$ & $\begin{array}{l}3 \\
3 \\
3 \\
3\end{array}$ & $\begin{array}{r}14.1 \\
23.0 \\
19.8 \\
6.4\end{array}$ & $\begin{array}{l}1.0 \\
1.1 \\
1.2 \\
0.7\end{array}$ & $\begin{array}{l}0.47 \\
0.56 \\
0.59 \\
0.44\end{array}$ & $\begin{array}{l}46.5 \\
38.4 \\
40.8 \\
50.4\end{array}$ & $\begin{array}{l}0.4 \\
0.4 \\
0.5 \\
0.4\end{array}$ & $\begin{array}{l}0.022 \\
0.017 \\
0.018 \\
0.041\end{array}$ & $\begin{array}{l}0.05 \\
0.05 \\
0.06 \\
0.04\end{array}$ & $\begin{array}{l}0.27 \\
0.29 \\
0.32 \\
0.49\end{array}$ & $\begin{array}{l}0.05 \mathrm{H} \\
0.08 \\
0.05 \mathrm{H} \\
0.07\end{array}$ & $\begin{array}{l}0.05 \mathrm{H} \\
0.05 \mathrm{H} \\
0.05 \mathrm{H} \\
0.05 \mathrm{H}\end{array}$ & $\begin{array}{l}38.02 \\
35.46 \\
35.88 \\
40.68\end{array}$ & $\begin{array}{r}100.93 \\
99.41 \\
99.27 \\
99.71\end{array}$ \\
\hline $\begin{array}{l}-6-7,20-22 \\
-6, C C \\
-1-7,90-92 \\
-7-6,100-120 \\
-8-1,120-122 \\
-8-1,120-122 b \\
-8-3,15-17 \\
-8-7,40-42 \\
-9-2,30-32 \\
-10-1,71-73 \\
-10-7,3-5 \\
-12-1,72-74 \\
-13-1,13-22 \\
-16-1,58-60 \\
-16, C C(4-7) \\
-19-1,23-25\end{array}$ & $\begin{array}{l}4 \\
4 \\
5 \\
5 \\
5 \\
5 \\
5 \\
5 \\
5 \\
5 \\
5 \\
5 \\
5 \\
5 \\
5 \\
5\end{array}$ & $\begin{array}{r}65.9 \\
40.0 \\
1.7 \\
3.0 \\
0.5 \\
5.1 \\
0.6 \\
0.7 \\
0.8 \\
1.8 \\
2.1 \\
1.2 \\
2.0 \\
1.6 \\
1.2 \\
2.7\end{array}$ & $\begin{array}{l}3.9 \\
9.2 \\
0.3 \\
0.7 \\
0.2 \mathrm{H} \\
0.4 \\
0.2 \mathrm{H} \\
0.2 \mathrm{H} \\
0.2 \mathrm{H} \\
0.5 \\
0.4 \\
0.2 \\
0.6 \\
0.5 \\
0.3 \\
0.7\end{array}$ & $\begin{array}{l}1.54 \\
2.23 \\
0.77 \\
0.84 \\
0.72 \\
0.12 \\
0.71 \\
0.71 \\
0.79 \\
0.86 \\
0.94 \\
0.85 \\
1.05 \\
0.96 \\
0.99 \\
1.08\end{array}$ & $\begin{array}{r}3.5 \\
14.8 \\
54.5 \\
52.3 \\
53.7 \\
2.3 \\
53.6 \\
52.6 \\
54.2 \\
52.0 \\
52.8 \\
54.7 \\
53.8 \\
52.9 \\
53.4 \\
50.9\end{array}$ & $\begin{array}{c}3.4 \\
8.6 \\
0.2 \\
0.8 \\
0.1 \mathrm{H} \\
59.5 \\
0.1 \\
0.1 \\
0.1 \\
0.4 \\
0.4 \\
0.2 \\
0.7 \\
0.7 \\
1.1 \\
1.2\end{array}$ & $\begin{array}{l}0.022 \\
0.049 \\
0.010 \mathrm{H} \\
0.010 \mathrm{H} \\
0.010 \mathrm{H} \\
0.060 \\
0.010 \mathrm{H} \\
0.010 \mathrm{H} \\
0.010 \mathrm{H} \\
0.010 \mathrm{H} \\
0.010 \mathrm{H} \\
0.010 \mathrm{H} \\
0.010 \mathrm{H} \\
0.012 \\
0.018 \\
0.098\end{array}$ & $\begin{array}{l}0.39 \\
1.07 \\
0.04 \\
0.13 \\
0.03 \\
0.04 \\
0.03 \\
0.03 \\
0.03 \\
0.10 \\
0.09 \\
0.04 \\
0.09 \\
0.09 \\
0.05 \\
0.12\end{array}$ & $\begin{array}{l}2.45 \\
1.46 \\
0.03 \\
0.05 \\
- \\
0.05 \\
- \\
- \\
- \\
- \\
- \\
- \\
- \\
- \\
- \\
-\end{array}$ & $\begin{array}{l}2.19 \\
2.26 \\
0.09 \\
0.08 \\
0.08 \\
0.15 \\
0.07 \\
0.07 \\
0.09 \\
0.08 \\
0.09 \\
0.10 \\
0.08 \\
0.08 \\
0.09 \\
0.15\end{array}$ & $\begin{array}{l}1.78 \\
2.63 \\
0.05 \mathrm{H} \\
0.05 \mathrm{H} \\
0.05 \mathrm{H} \\
0.15 \\
0.05 \mathrm{H} \\
0.05 \mathrm{H} \\
0.05 \mathrm{H} \\
0.05 \mathrm{H} \\
0.05 \mathrm{H} \\
0.05 \mathrm{H} \\
0.05 \mathrm{H} \\
0.05 \mathrm{H} \\
0.05 \mathrm{H} \\
0.06\end{array}$ & $\begin{array}{l}10.95 \\
11.76 \\
43.02 \\
41.63 \\
43.83 \\
32.47 \\
43.71 \\
43.93 \\
43.60 \\
42.86 \\
43.04 \\
43.25 \\
41.99 \\
41.83 \\
42.65 \\
41.88\end{array}$ & $\begin{array}{r}96.02 \\
94.06 \\
100.71 \\
99.56 \\
99.09 \\
100.34 \\
99.04 \\
98.35 \\
99.87 \\
98.66 \\
99.92 \\
100.60 \\
100.36 \\
98.71 \\
99.87 \\
98.89\end{array}$ \\
\hline $\begin{array}{c}433 \mathrm{~B}-1-4,119-120 \\
-1-4,120-126 \\
-2-1,0-150 \\
-3-1,20-60\end{array}$ & $\begin{array}{l}5 \\
5 \\
5 \\
5\end{array}$ & $\begin{array}{l}1.6 \\
1.5 \\
1.8 \\
0.9\end{array}$ & $\begin{array}{l}0.5 \\
0.5 \\
0.6 \\
0.3\end{array}$ & $\begin{array}{l}0.92 \\
0.93 \\
0.94 \\
0.96\end{array}$ & $\begin{array}{l}51.9 \\
53.3 \\
52.9 \\
53.6\end{array}$ & $\begin{array}{l}0.5 \\
0.6 \\
0.7 \\
0.5\end{array}$ & $\begin{array}{l}0.010 \mathrm{H} \\
0.011 \\
0.014 \\
0.026\end{array}$ & $\begin{array}{l}0.08 \\
0.08 \\
0.10 \\
0.05\end{array}$ & $\begin{array}{l}- \\
- \\
- \\
-\end{array}$ & $\begin{array}{l}0.06 \\
0.06 \\
0.06 \\
0.07\end{array}$ & $\begin{array}{l}0.05 \mathrm{H} \\
0.05 \mathrm{H} \\
0.05 \mathrm{H} \\
0.05 \mathrm{H}\end{array}$ & $\begin{array}{l}43.27 \\
42.26 \\
42.07 \\
43.03\end{array}$ & $\begin{array}{l}98.89 \\
99.29 \\
99.23 \\
99.53\end{array}$ \\
\hline $433 C-3-2,103-106$ & 6 & 20.3 & 5.9 & 2.58 & 34.7 & 6.6 & 0.116 & 1.23 & 0.02 & 1.24 & 0.45 & 26.94 & 100.07 \\
\hline
\end{tabular}

Note: Total iron and manganese are calculated as $\mathrm{Fe}_{2} \mathrm{O}_{3}$ and $\mathrm{Mn}_{3} \mathrm{O}_{4}$; Ign is ignition loss at $1000^{\circ} \mathrm{C} .433 \mathrm{~A}-6-7,20-22 \mathrm{~cm}: \mathrm{P}_{2} \mathrm{O}_{5}=1.0 \%$; $433 \mathrm{~A}-6, \mathrm{CC}: \mathrm{P}_{2} \mathrm{O}_{5}=3.4 \%$.

centration is higher than that of the detrital volcanic material, when compared with the concentration in the first underlying basalt flow (chemical analysis from shipboard report). The reef sequence (Units 5 ana 6) is characterized by a low Sr content. In shallow water, strontium is removed from sea water by calcareous organisms, specifically the coral-oölite-algae association (Turekian, 1964; Bathurst, 1971). It appears that the Sr-impoverishment of the reef facies is a result of diagenesis. The diagenetic evolution of these shallowwater carbonates is discussed by McKenzie et al. (this volume). They describe the dissolution of aragonite and formation of blocky calcite and zeolites in the upper part of the sequence. Thus, the strontium moves to the overlying volcaniclastic deposits, and is retained in the authigenic zeolites, barite, or carbonate-apatite of this formation.

\section{EVOLUTION ALONG THE SEDIMENTARY SEQUENCE AT SITE 433: INFLUENCES OF VOLCANISM, SUBSIDENCE, HIATUS, AND ACCUMULATION RATES}

The mineralogical and geochemical data, compared with the biostratigraphic results, allow construction of a 
TABLE 3

Chemical Analyses of Sedimentary Depostis from Site 433, Leg 55, Suiko Seamount. Trace Elements (ppm)

\begin{tabular}{|c|c|c|c|c|c|c|c|c|c|c|c|c|}
\hline $\begin{array}{c}\text { Sample } \\
\text { (Interval in } \mathrm{cm} \text { ) }\end{array}$ & Unit & $\mathrm{Sr}$ & $\mathrm{Ba}$ & V & $\mathrm{Ni}$ & Co & $\mathrm{Cr}$ & B & $\mathrm{Zn}$ & $\mathrm{Ga}$ & $\mathrm{Cu}$ & $\mathrm{Pb}$ \\
\hline $433-1-1,60-62$ & 1 & 1104 & 676 & 74 & 30 & 11 & 43 & 72 & 114 & 11 & 26 & 36 \\
\hline$-1-1,129-131$ & 1 & 1402 & 1308 & 75 & 33 & 15 & 49 & 42 & 123 & 12 & 23 & 37 \\
\hline $433 \mathrm{~A}-1-1,7-9$ & 1 & 890 & 1025 & 61 & 28 & 17 & 53 & 57 & 84 & 7 & 18 & 139 \\
\hline$-2-1,70-72$ & 1 & 933 & 1013 & 106 & 38 & 22 & 64 & 73 & 124 & 16 & 18 & 25 \\
\hline$-3-1,80-85$ & 1 & 2015 & 841 & $5 \mathrm{H}$ & 2 & $2 \mathrm{H}$ & $5 \mathrm{H}$ & 13 & $4 \mathrm{H}$ & $2 \mathrm{H}$ & $2 \mathrm{H}$ & $2 \mathrm{H}$ \\
\hline$-3-6,21-23$ & 1 & 2151 & 818 & $5 \mathrm{H}$ & 3 & $2 \mathrm{H}$ & $5 \mathrm{H}$ & 24 & 8 & $2 \mathrm{H}$ & 2 & $2 \mathrm{H}$ \\
\hline $433-1-2,40-42$ & 2 & 1368 & 1803 & 101 & 63 & 34 & 80 & 80 & 192 & 17 & 25 & 28 \\
\hline$-1-2,127-129$ & 2 & 2436 & 1937 & $5 \mathrm{H}$ & 21 & 6 & 9 & 52 & 38 & 3 & 8 & 13 \\
\hline$-1-3,7-9$ & 2 & 2217 & 1532 & 42 & 38 & 8 & 34 & 79 & 95 & 6 & 26 & 24 \\
\hline$-1-3,104-106$ & 2 & 2127 & 1967 & 35 & 46 & 32 & 18 & 68 & 68 & 3 & 12 & 29 \\
\hline$-1-4,26-28$ & 2 & $3084 \mathrm{E}$ & 1867 & 33 & 20 & 35 & 7 & 68 & 33 & 3 & 3 & 23 \\
\hline $433 \mathrm{~A}-4-1,7-8$ & 2 & 2710 & 2683 & 17 & 22 & 33 & 27 & 71 & 102 & 4 & 6 & 17 \\
\hline$-4-2,66-68$ & 2 & 678 & 4570 & 107 & 74 & 47 & 72 & 157 & 112 & 13 & 49 & 99 \\
\hline$-4-2,68-70$ & 2 & 561 & 3971 & 102 & 77 & 49 & 80 & 152 & 122 & 14 & 43 & 63 \\
\hline$-4-2,100-105$ & 2 & 1029 & 3763 & 64 & 46 & 11 & 30 & 115 & 57 & 6 & 47 & 67 \\
\hline$-4-3,55-60$ & 2 & 1396 & 3180 & 24 & 33 & 31 & 16 & 135 & 62 & 4 & 18 & 26 \\
\hline$-4-3,85-90$ & 2 & 2115 & 3042 & 16 & 32 & 22 & 10 & 103 & 71 & 4 & 8 & 16 \\
\hline$-4-4,60-62$ & 2 & 2309 & 3123 & 24 & 29 & 17 & 22 & 96 & 113 & 6 & 0 & 20 \\
\hline$-4-6,92-95$ & 2 & 2586 & 1958 & 14 & 17 & 10 & $5 \mathrm{H}$ & 50 & 55 & 5 & 9 & 22 \\
\hline$-5-2,130-132$ & 2 & 2598 & 1074 & $5 \mathrm{H}$ & 7 & $2 \mathrm{H}$ & $5 \mathrm{H}$ & 28 & 2 & 2 & $2 \mathrm{H}$ & 2 \\
\hline$-5-4,20-22$ & 2 & 2705 & 2099 & $5 \mathrm{H}$ & 19 & $2 \mathrm{H}$ & $5 \mathrm{H}$ & 37 & 13 & 2 & $2 \mathrm{H}$ & $2 \mathrm{H}$ \\
\hline$-5-5,110-112$ & 2 & $3258 \mathrm{E}$ & 4549 & 47 & 32 & 36 & 14 & 51 & 72 & 6 & 6 & 17 \\
\hline$-6-1,50-53$ & 3 & $3014 \mathrm{E}$ & 3015 & $5 \mathrm{H}$ & 17 & $2 \mathrm{H}$ & $5 \mathrm{H}$ & $5 \mathrm{H}$ & 23 & 2 & $2 \mathrm{H}$ & $2 \mathrm{H}$ \\
\hline$-6-3,140-144$ & 3 & 2441 & 3709 & 10 & 21 & $2 \mathrm{H}$ & $5 \mathrm{H}$ & 33 & 56 & 3 & 3 & 6 \\
\hline$-6-6,60-62$ & 3 & $3338 \mathrm{E}$ & 3314 & 36 & 29 & 38 & 30 & 75 & 11 & 3 & $2 \mathrm{H}$ & 22 \\
\hline$-6-6,120-122$ & 3 & $3822 \mathrm{E}$ & $6213 \mathrm{E}$ & 33 & 19 & 38 & 13 & $5 \mathrm{H}$ & 13 & 4 & 2 & 11 \\
\hline$-6-7,20-22$ & 4 & 908 & S & 76 & 104 & 38 & 94 & 266 & 60 & 13 & 58 & 36 \\
\hline$-6, \mathrm{CC}$ & 4 & 1178 & S & 173 & 107 & 38 & 270 & 178 & 176 & 22 & 83 & 20 \\
\hline$-1-7,90-92$ & 5 & 777 & 249 & 9 & 7 & $2 \mathrm{H}$ & 15 & 20 & 19 & 3 & 2 & $2 \mathrm{H}$ \\
\hline$-7-6,100-120$ & 5 & 624 & 364 & 26 & 12 & $2 \mathrm{H}$ & 34 & 15 & 24 & 2 & 3 & $2 \mathrm{H}$ \\
\hline$-8-1,120-122$ & 5 & 680 & 9 & 9 & 10 & 3 & 14 & 25 & 8 & $2 \mathrm{H}$ & 42 & 10 \\
\hline$-8-1,120-122 b$ & 5 & 33 & 293 & $5 \mathrm{H}$ & 107 & $2 \mathrm{H}$ & 7 & $5 \mathrm{H}$ & $2 \mathrm{H}$ & $2 \mathrm{H}$ & 16 & 4 \\
\hline$-8-3,15-17$ & 5 & 609 & 10 & 8 & 19 & $2 \mathrm{H}$ & 48 & $5 \mathrm{H}$ & 10 & 3 & 4 & $2 \mathrm{H}$ \\
\hline$-8-7,40-42$ & 5 & 602 & 8 & 22 & 9 & $2 \mathrm{H}$ & 16 & $5 \mathrm{H}$ & 25 & 5 & 5 & 5 \\
\hline$-9-2,30-32$ & 5 & 574 & 45 & $5 \mathrm{H}$ & 7 & $2 \mathrm{H}$ & 8 & $5 \mathrm{H}$ & 9 & $2 \mathrm{H}$ & 2 & 67 \\
\hline$-10-1,71-73$ & 5 & 818 & 7 & 42 & 13 & 31 & 25 & 21 & 24 & 4 & 6 & 5 \\
\hline$-10-7,3-5$ & 5 & 755 & 6 & 16 & 3 & $2 \mathrm{H}$ & 19 & 26 & 17 & 3 & 4 & $2 \mathrm{H}$ \\
\hline$-12-1,72-74$ & 5 & 613 & 12 & 6 & 9 & $2 \mathrm{H}$ & 13 & 13 & 11 & 4 & 4 & 26 \\
\hline$-13-1,20-22$ & 5 & 811 & 17 & 5 & 9 & $2 \mathrm{H}$ & 20 & $5 \mathrm{H}$ & 16 & 2 & 6 & 76 \\
\hline$-16-1,58-60$ & 5 & 757 & 23 & 16 & 13 & $2 \mathrm{H}$ & 21 & $5 \mathrm{H}$ & 16 & 3 & 11 & 80 \\
\hline$-16, \mathrm{CC}(4-7)$ & 5 & 695 & 101 & 9 & 13 & $2 \mathrm{H}$ & 35 & 30 & 18 & 3 & 4 & 31 \\
\hline$-19-1,23-25$ & 5 & 497 & 26 & 6 & 8 & $2 \mathrm{H}$ & 27 & $5 \mathrm{H}$ & 17 & $2 \mathrm{H}$ & 10 & 7 \\
\hline 433B-1-4, 119-120 & 5 & 715 & 16 & $5 \mathrm{H}$ & 15 & $2 \mathrm{H}$ & 16 & $5 \mathrm{H}$ & 12 & 2 & 4 & 20 \\
\hline$-1-4,120-126$ & 5 & 613 & 29 & $5 \mathrm{H}$ & 10 & $2 \mathrm{H}$ & 14 & $5 \mathrm{H}$ & 2 & $2 \mathrm{H}$ & 4 & 11 \\
\hline$-2-1,0-150$ & 5 & 543 & 19 & $5 \mathrm{H}$ & 11 & $2 \mathrm{H}$ & 15 & $5 \mathrm{H}$ & 3 & $2 \mathrm{H}$ & 2 & 57 \\
\hline$-3-1,20-60$ & 5 & 488 & 10 & $5 \mathrm{H}$ & $2 \mathrm{H}$ & $2 \mathrm{H}$ & $5 \mathrm{H}$ & $5 \mathrm{H}$ & $2 \mathrm{H}$ & $2 \mathrm{H}$ & 4 & 20 \\
\hline $433 C-3-2,103-106$ & 6 & 575 & 103 & 163 & 40 & 13 & 63 & 21 & 100 & 11 & 24 & 27 \\
\hline
\end{tabular}

Note: $\mathrm{H}$ : value below the lower detection limit; $\mathrm{S}$ : value over the upper detection limit; $\mathrm{E}$ : extrapolated value.

depositional history of the Suiko seamount sedimentary sequence.

\section{The Paleocene Shallow Warm Water Sedimentation}

The reef calcareous sand lies on the eroded surface of an alkalic basalt flow, and ranges upward to a carbonate mud. In the sequence the diagenesis described by McKenzie et al. (this volume) has occurred, as the constant volcanic influence induced the dissolution of aragonite and the formation of calcite and zeolites. The occurrence of diagenetic zeolites in sedimentary rocks is common in a volcanic environment (Coombs et al.,
1959; Staples, 1965; Sheppard and Gude, 1973), but is generally related to the alteration of volcanic glass enclosed in the deposits (Hay, 1966; Cronan, 1974; Hoffert et al., 1978).

Another authigenic mineral in the upper part of the lagoonal sequence is pyrite, which occurs in two forms, framboids (Plate 1) in the pores of reef organism fragments and massive concretions of centimeter size.

Authigenic pyrite is a well-known constituent of sediments, whose geographic and stratigraphic occurrences were reviewed by Love and Amstutz in 1966, and since that time it has often been observed (Hein and 


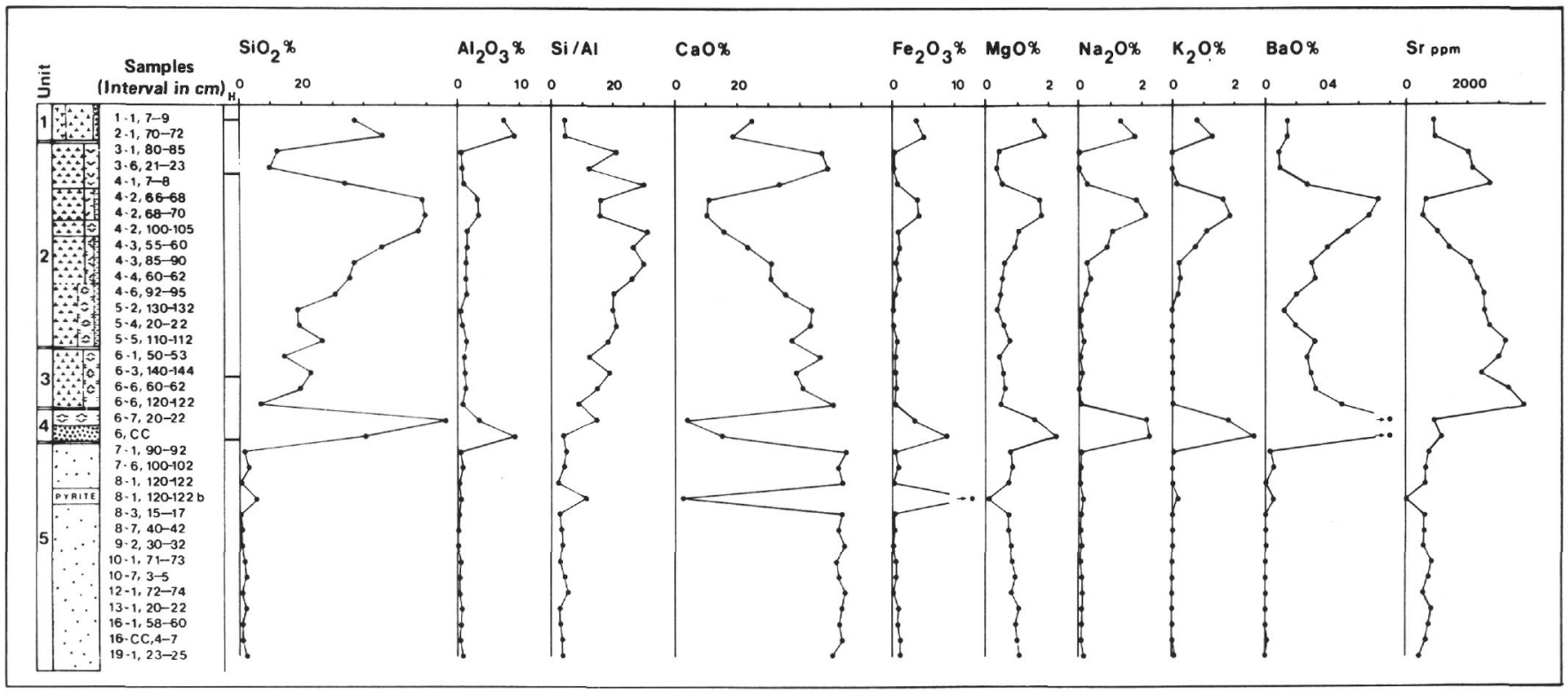

A

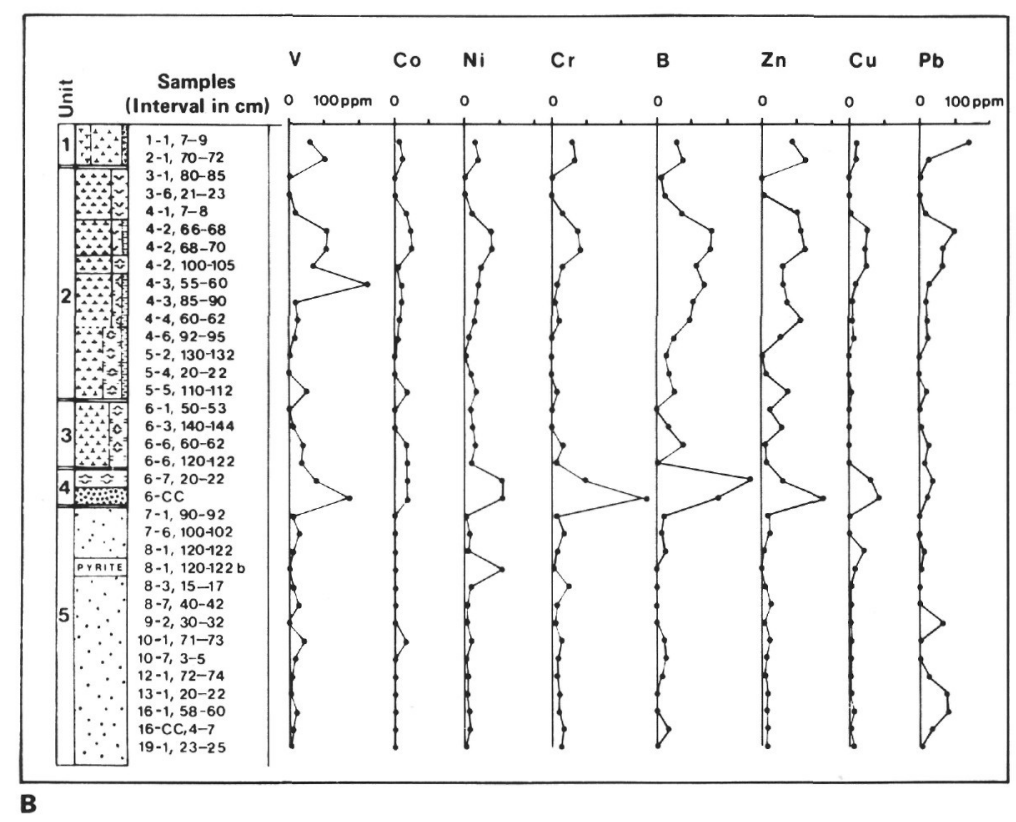

Figure 1. (A) Variations in contents of major elements (\%) and $\mathrm{Sr}(\mathrm{ppm})$ and in Si/Al ratio along the sedimentary column of Hole $433 \mathrm{~A}$. The thickness is not to scale. (B) Variations in trace-element contents (ppm) along the sedimentary column of Hole $433 A$.

Griggs, 1972; Marchand, 1974; Odent and Prouvost, 1974; Dell, 1975). Organic activity in a reducing environment is the commonly hypothesized mechanism for framboidal pyrite formation; different explanations of the process are given by Rickard (1970); Sweeney and Kaplan (1973); and Goldhaber and Kaplan (1974). High organic content of the calcareous deposits is also responsible for the very small amount of gypsum present (Arrhenius, 1963; Siesser and Rogers, 1976; Briskin and Schreiber, 1978).

The source of $\mathrm{S}$ and $\mathrm{SO}_{4}{ }^{2-}$ could be steams migrating through the volcanic terrace. This volcanic influence promotes the formation of the nickel-rich pyrite concretions. The occurrence of pyrite in zones of high vol- canic activity has been described by Goldhaber and Kaplan (1974) and by Bonatti et al. (1976).

The association of zeolite, pyrite, and scarce gypsum in the lagoonal deposits appears to be a result of simultaneous organic and volcanic influence in shallow warm water environment.

\section{The Period of the Miocene Hiatus}

At the end of the Paleocene, a period of nonsedimentation began, and the Eocene and Oligocene are not represented in the section. In the interval below the upper Miocene pelagic sediment and overlying the lagoonal formation, the sedimentary deposits are composed of a volcanic mud and siliceous ooze (Unit 4) and 
TABLE 4

Element-Ratios in Sedimentary Deposits from Site 433

\begin{tabular}{|c|c|c|c|c|c|}
\hline $\begin{array}{c}\text { Sample } \\
\text { (Interval in } \mathrm{cm} \text { ) }\end{array}$ & Unit & $\mathrm{Si} / \mathrm{Al}$ & $\mathrm{Mn} / \mathrm{Fe}$ & $\mathrm{Fe} / \mathrm{Ti}$ & $\frac{\mathrm{Sr}}{\mathrm{Ca}} \times 10^{3}$ \\
\hline $\begin{array}{l}433-1-1,60-62 \\
-1-1,129-131\end{array}$ & $\begin{array}{l}1 \\
1\end{array}$ & $\begin{array}{l}6.86 \\
4.20\end{array}$ & $\begin{array}{l}19.6 \\
14.2\end{array}$ & $\begin{array}{r}9.67 \\
11.27\end{array}$ & $\begin{array}{l}5.62 \\
7.52\end{array}$ \\
\hline $\begin{array}{r}433 \mathrm{~A}-1-1,7-9 \\
-2-1,70-72 \\
-3-1,80-85 \\
-3-6,21-23\end{array}$ & $\begin{array}{l}1 \\
1 \\
1 \\
1\end{array}$ & $\begin{array}{r}4.38 \\
4.33 \\
21.12 \\
12.32\end{array}$ & $\begin{array}{l}15.3 \\
13.1 \\
61.8 \\
66.9\end{array}$ & $\begin{array}{r}11.29 \\
12.37 \\
7.73 \\
7.73\end{array}$ & $\begin{array}{l}5.11 \\
7.06 \\
6.00 \\
6.14\end{array}$ \\
\hline $\begin{array}{c}433-1-2,40-42 \\
-1-2,127-129 \\
-1-3,7-9 \\
-1-3,104-106 \\
-1-4,26-28\end{array}$ & $\begin{array}{l}2 \\
2 \\
2 \\
2 \\
2\end{array}$ & $\begin{array}{r}5.75 \\
39.31 \\
9.85 \\
14.55 \\
50.16\end{array}$ & $\begin{array}{r}10.3 \\
25.7 \\
12.6 \\
16.8 \\
103.0\end{array}$ & $\begin{array}{r}14.42 \\
11.60 \\
13.05 \\
15.95 \\
5.80\end{array}$ & $\begin{array}{r}9.63 \\
9.10 \\
9.98 \\
8.51 \\
11.87\end{array}$ \\
\hline $\begin{array}{c}433 \mathrm{~A}-4-1,7-8 \\
-4-2,66-68 \\
-4-2,68-70 \\
-4-2,100-105 \\
-4-3,55-60 \\
-4-3,85-90 \\
-4-4,60-62 \\
-4-6,92-95 \\
-5-2,130-132 \\
-5-4,20-22 \\
-5-5,110-112\end{array}$ & $\begin{array}{l}2 \\
2 \\
2 \\
2 \\
2 \\
2 \\
2 \\
2 \\
2 \\
2 \\
2\end{array}$ & $\begin{array}{l}29.74 \\
16.20 \\
15.35 \\
31.46 \\
26.58 \\
29.36 \\
25.96 \\
20.51 \\
19.36 \\
21.12 \\
18.07\end{array}$ & $\begin{array}{c}32.6 \\
6.16 \\
6.23 \\
11.5 \\
24.7 \\
30.9 \\
28.96 \\
45.3 \\
103.0 \\
41.12 \\
24.0\end{array}$ & $\begin{array}{r}13.92 \\
25.25 \\
27.71 \\
13.05 \\
12.89 \\
11.60 \\
15.47 \\
11.60 \\
7.73 \\
8.70 \\
9.94\end{array}$ & $\begin{array}{r}11.43 \\
8.87 \\
7.86 \\
9.12 \\
8.32 \\
9.65 \\
10.67 \\
10.29 \\
8.31 \\
8.75 \\
12.30\end{array}$ \\
\hline $\begin{array}{l}-6-1,50-53 \\
-6-3,140-144 \\
-6-6,60-62 \\
-6-6,120-122\end{array}$ & $\begin{array}{l}3 \\
3 \\
3 \\
3\end{array}$ & $\begin{array}{r}12.41 \\
18.40 \\
14.52 \\
8.05\end{array}$ & $\begin{array}{r}56.6 \\
43.7 \\
37.0 \\
105.5\end{array}$ & $\begin{array}{r}9.28 \\
9.28 \\
9.67 \\
11.60\end{array}$ & $\begin{array}{r}9.08 \\
8.90 \\
11.46 \\
10.62\end{array}$ \\
\hline $\begin{array}{l}-6-7,20-22 \\
-6, \mathrm{CC}\end{array}$ & $\begin{array}{l}4 \\
4\end{array}$ & $\begin{array}{r}14.87 \\
3.83\end{array}$ & $\begin{array}{l}6.7 \\
5.8\end{array}$ & $\begin{array}{r}10.11 \\
9.32\end{array}$ & $\begin{array}{l}36.33 \\
11.15\end{array}$ \\
\hline $\begin{array}{l}-1-7,90-92 \\
-7-6,100-120 \\
-8-1,120-122 \\
-8-1,120-122 b \\
-8-3,15-17 \\
-8-7,40-42 \\
-9-2,30-32 \\
-10-1,71-73 \\
-10-7,3-5, \\
-12-1,72-74 \\
-13-1,20-22 \\
-16-1,58-60 \\
-16, C C(4-7) \\
-19-1,23-25\end{array}$ & $\begin{array}{l}5 \\
5 \\
5 \\
5 \\
5 \\
5 \\
5 \\
5 \\
5 \\
5 \\
5 \\
5 \\
5 \\
5\end{array}$ & $\begin{array}{r}4.99 \\
3.77 \\
4.20 \\
11.22 \\
2.64 \\
3.08 \\
3.52 \\
3.17 \\
4.62 \\
5.28 \\
2.93 \\
2.82 \\
3.52 \\
3.39\end{array}$ & $\begin{array}{r}51.6 \\
12.8 \\
103.0 \\
104.0 \\
103.0 \\
103.0 \\
103.0 \\
25.7 \\
25.7 \\
51.5 \\
14.6 \\
17.6 \\
16.9 \\
84.1\end{array}$ & $\begin{array}{r}5.80 \\
7.14 \\
3.87 \\
1725.50 \\
3.87 \\
3.87 \\
3.87 \\
4.64 \\
5.16 \\
5.80 \\
9.02 \\
9.02 \\
25.52 \\
11.60\end{array}$ & $\begin{array}{l}1.99 \\
1.67 \\
1.77 \\
2.01 \\
1.59 \\
1.60 \\
1.48 \\
2.20 \\
2.00 \\
1.57 \\
2.11 \\
2.00 \\
1.82 \\
1.37\end{array}$ \\
\hline $\begin{array}{c}433 \mathrm{~B}-1-4,119-120 \\
-1-4,120-126 \\
-2-1,0-150 \\
-3-1,20-60\end{array}$ & $\begin{array}{l}5 \\
5 \\
5 \\
5\end{array}$ & $\begin{array}{l}2.82 \\
2.64 \\
2.64 \\
2.64\end{array}$ & $\begin{array}{l}20.6 \\
18.9 \\
20.6 \\
53.6\end{array}$ & $\begin{array}{r}7.25 \\
8.70 \\
8.12 \\
11.60\end{array}$ & $\begin{array}{l}1.93 \\
1.61 \\
1.44 \\
1.27\end{array}$ \\
\hline $433 C-3-2,103-106$ & 6 & 3.03 & 18.10 & 6.22 & 2.32 \\
\hline
\end{tabular}

a calcareous ooze, in the lower part of Unit 3, which contains pyrite concretions. These formations appear to be lower Miocene, but show considerable reworking and contain organisms of diversified age.

\section{The Two Levels of Lithologic Unit 4}

The mineralogical and geochemical data show that the volcanic layer, in Unit 4, contains barite, apatite, and zeolites associated with plagioclases, quartz, detrital volcanic minerals, and calcareous components. This layer is enriched in barium and strontium.

The scanning-electron microscope photographs (Plate 2) show the evolution of the mineral assemblage in the coarse fraction. The feldspars are often well preserved, and their low degree of alteration consists of exfoliated

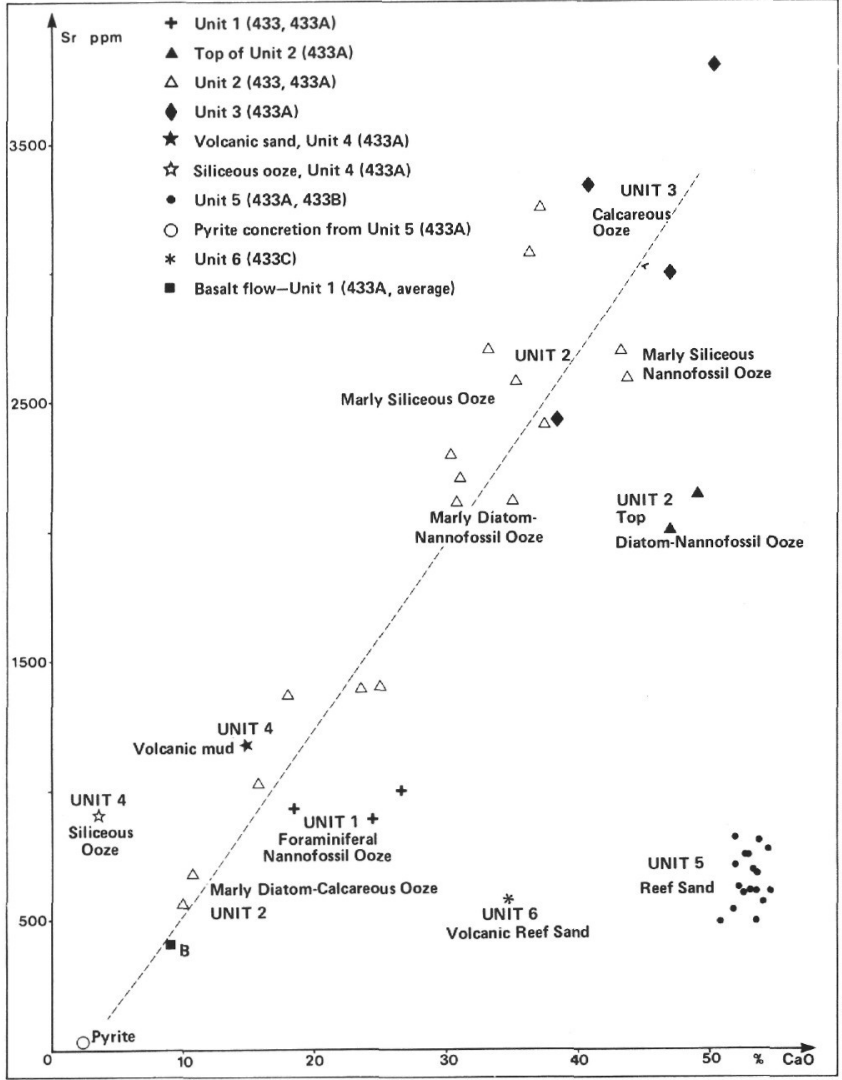

Figure 2. Relation between $\mathrm{CaO}(\%)$ and $\mathrm{Sr}(\mathrm{ppm})$ contents in bulk sediments from Site 433 (Holes 433, $433 A, 433 B, 433 C$ ).

fine sheets (figure 1). The volcanic debris occurs as rounded grains of volcanic glass, in which phenocrysts are associated with authigenic components as twinned crystals of zeolites (figures 2 and 3). They have been progressively coated by clays, calcareous debris (coccoliths), carbonate-apatite, barite, and secondary zeolites (figure 4). Figures 5, 6, 7, and 8 show these diversified components overlying a twinned crystal of zeolite. The barite crystals are very small and enclosed in the clays and phosphate matrix. Within the coating the nannofossil fragments are altered. In the pores, a very well crystallized secondary zeolite is associated with clays. Finally, the primary twinned crystals of zeolite are completely coated and form rounded indurated particles (figure 9).

These observations indicate that the tuffaceous level is a volcanic sand deposited in a marine environment and show its evolution to an indurated and consolidated layer. Such phenomena of alteration of volcanic sand and induration by authigenic processes in shallow sea water containing calcareous organisms are not unknown. Indurated sedimentary layers have been described by Morgenstein (1967) and by Hoffert et al. (1978), with reference to the same conditions, in shallow warm water.

Nevertheless, in the altered volcanic layer at Suiko Seamount the cementation processes have not formed a continuous consolidated layer with the final deposition 
of ferromanganese oxides. The high-activity environment maintained the sandy facies. The occurrence of carbonate-apatite and barite, and the high $\mathrm{Sr}$ contents of the authigenic minerals, also differentiate this tuffaceous layer from the indurated sediments from the South Pacific, and bring to mind the cemented formations from continental margins and seamounts of the Atlantic Ocean (Tooms et al., 1969; Le Lann, 1972; Karpoff et al., in preparation).

The occurrence of barite is reviewed by Cronan (1974); its formation under volcanic or shallow hydrothermal coastal environment and in sediments rich in organic material is cited by Goldberg et al. (1969), Sakai (1971), and by Dean and Schreiber (1978).

The volcanic sandy deposits have been altered and the authigenic process developed in relatively confined shallow water, in an active environment and under volcanic influence in the restricted basin on Suiko Seamount.

The upper part of Unit 4 is a siliceous ooze which has the same mineral and geochemical assemblages, with different ratios, as the underlying tuffaceous layer. The siliceous fauna comprises radiolarians ranging from lower Miocene to Pliocene. The reworked nannofossils are lower Miocene (Scientific staff, this volume; Schaff, personal communication).

The highly reworked character of Unit 4 and the mineral and planktonic assemblages allow me to interpret the two levels of this unit as resulting from grainsize and mechanical sorting of mixed volcanic and biogenic deposits.

\section{Evolution of Unit 4}

Thus, from the late Paleocene until the Miocene, sedimentation in our small graben basin on Suiko Seamount was very irregular. The hiatus at the end of lagoonal sedimentation corresponds to a period of nonsedimentation.

The onset of the pelagic sedimentation started with deposition of volcanic fine sand and pelagic material, probably introduced into the basin by currents during a period of tectonic activity and irregular, intermittent submergence of the seamount.

The high-energy environment induced a sorting of the biogenic and detrital material. The volcanic deposits were altered, with authigenesis taking place probably at the same time as the diagenetic evolution of the underlying lagoonal deposits. The contribution of components coming from the diagenetic reef formation, from volcanic steam, from the altered volcaniclastic material, and from the degraded organic matter, promoted authigenesis of silicates, sulfates, and carbonates.

The deposition of fresh volcaniclastic particles and reworked planktonic organisms was continuous during the early Miocene. This material was mixed with the older altered and authigenic components, which started to form an indurated sedimentary level in shallow warm-water environment.

At the top of the lower Miocene, the sedimentary deposits are more calcareous, and form the lower part of lithologic Unit 3. Near its top, this pelagic section contains pyrite concretions formed under volcanic influence, similar to those of the lagoonal deposits.

This sequence corresponds to a phase intermediate between the high-energy depositional environment with volcanic influences (Unit 4) and the pelagic sedimentation of Unit 2. The accumulation rate was vey high during this period, and the shallow water became cooler. The hiatus occurring at middle Miocene can be interpreted as non-sedimentation during the subsidence of Suiko Seamount, and a period of tectonic activity in the basin.

\section{The Period of Pelagic Sedimentation}

After the middle Miocene hiatus, sediment accumulation resumed with formation of siliceous-calcareous deposits. The increase in siliceous components and the decrease of calcareous organisms from Unit 3 to Unit 2 are gradual. The sediment accumulation rate was very high $(5 \mathrm{~cm} / 1000$ years), and the material has been reworked. A change in the environment shown by the fauna assemblage (Butt, this volume) is due to the gradual cooling of the water mass.

The change in the siliceous/calcareous biogenic ratio is a result of the increase in depth of the sedimentation to deeper and cooler water. Under the hypothesis of a lysocline higher than the actual $C C D$, carbonate material would be selectively dissolved. Nevertheless, the grading can also be the result of reworking and sorting in an active environment.

The geochemical data indicate that the lower Pliocene hiatus, located in the upper part of Unit 2, started early in the sedimentary history with a recrudescence of calcareous deposits. The authigenic processes are also indicated by the increase in boron within clays above the hiatus.

The lower Pliocene hiatus marks a sharp change in the depositional environment to a more typical recent siliceous calcareous pelagic deposit. The upper two Units, 2 and 1, are distinguished by their sedimentological constitution, and the last hiatus during the late Pliocene did not induce geochemical or mineralogical changes in Unit 1. The top of the sedimentary column is a typical Recent pelagic deposit.

\section{CONCLUSION}

The sedimentary depositional history at Suiko Seamount comprises three major periods during the subsidence of the seamount: (1) The reef period, during which the calcareous lagoonal deposits had a diagenetic evolution, finishes at the upper Paleocene. The environment involved shallow warm water, volcanic steam influence, and a confined basin. (2) Deposition of a detrital volcanic sand mixed with siliceous biogenic material. The authigenetic process started producing an indurated level. The environment was characterized by high-energy reworking, sorting, volcanic influence, shallow warm water in a restricted basin. (3) Pelagic sedimentation in progressively cooler environment started in the late Miocene. The sediments are reworked up to the upper Pliocene. 
The depositional environment and the sedimentary sequence at Site 433 on Suiko Seamount differ from those of the other seamounts of the Emperor chain. This is a result of the location of the studied site in a tectonic basin within the lateral lagoon of the seamount. This situation induces sluggish exchange with the open sea. The tectonic activity and intermittent pulses during the subsidence and submergence of the island favored reworking of the successive deposits, and gave rise to periods of non-sedimentation. In the meantime, diagenetic and authigenetic phenomena transformed the sedimentary deposits.

\section{ACKNOWLEDGMENTS}

For the many helpful discussions during and after Leg 55, I thank Judith McKenzie, and, in Strasbourg, André Schaaf.

The analytical work was carried out with the technical support of the Institut de Géologie (Strasbourg, France). The S. E. M. microphotographs are the work of Irène Peterschmitt.

\section{REFERENCES}

Arrhenius, G., 1963. Pelagic sediments. In Hill, M. N. (Ed.), The Sea: New York (Interscience), v. 3, pp. 655-727.

Bathurst, R. G. C., 1971. Carbonate sediments and their diagenesis, Developments in Sedimentology, Elsevier Pub., v. 12, p. 620.

Berner, R. A., 1970. Sedimentary pyrite formation, Am. J. Sci., v. 268, pp. 1-23.

Boström, K., Joensuu, O., Moore, C., Boström, B., Dalziel, M., and Horowitz, A., 1973. Geochemistry of barium in pelagic sediments, Lithos, v. 6, pp. 159-174.

Bonatti, E., Honnorez-Guerstein, M. B., Honnorez, J., and Stern, C., 1976. Hydrothermal pyrite concretions from the Romanche Trench (equatorial Atlantic): metallogenesis in oceanic fractures zones, Earth Plan. Sci. Letters, v. 32, pp. $1-10$.

Briskin, M. and Schreiber, B. C., 1978. Authigenic gypsum in marine sediments, Mar. Geol., v. 28, pp. 37-50.

Brongersma-Sanders, M., 1967. Barium in pelagic sediments and diatoms, K. K. L. Nederl. Akad. Wetersh. Proc., B, pp. 93-99.

Chow, T. J. and Goldberg, E. D., 1960. On the marine geochemistry of barium, Geochim. Cosmochim. Acta, v. 20, pp. 192-198.

Coombs, D. S., Ellis, A. J., Fyfe, W. S., and Taylor, A. M., 1959. The zeolite facies with comments on the interpretation to hydrothermal syntheses, Geochim. Cosmochim. Acta., v. 17, pp. 53-107.

Cronan, D. S., 1974. Authigenic minerals in deep sea sediments. In Hill, M. N., (Ed.), The Sea: New York, (Interscience), v. 5, pp. 491-525.

Dean, W. E. and Schreiber, B. C., 1978. Authigenic barite, Leg 41. In Lancelot, Y., Seibold, E., et al., Initial Reports of the Deep Sea Drilling Project, v. 41: Washington (U. S. Government Printing Office), pp. 915-931.

Dell, C. I., 1975. Pyrite concretions in sediments from South Bay,. Lake Huron, Can. J. Earth Sc., v. 12, pp. 1077-1083.

El Wakeel, S. K. and Riley, J. P., 1961. Chemical and mineralogical studies of deep sea sediments, Geochim. Cosmochim. Acta, v. 25, pp. 110-146.
Goldberg, E. D. and Arrhenius, G., 1958. Chemistry of pelagic sediments, Geochim. Cosmochim. Acta, v. 13, pp. 153212.

Goldberg, E. D., Somayajulu, B. L. K., and Galloway, J., 1969. Differences between barites of marine and continental origins, Geochim. Cosmochim. Acta, v. 33, pp. 287-289.

Goldhaber, M. B. and Kaplan, I. R., 1974. The sulfur cycle. In Hill, M. N. (Ed.), The Sea: New York (Interscience), v. 5, pp. 569-655.

Hay, R. L., 1966. Zeolites and zeolitic reactions in sedimentary rocks, Geol. Soc. Amer. Spec. Paper, v. 85, p. 130.

Hein, J. R. and Griggs, G. B., 1972. Distribution and scanning electron microscope (S. E. M.) observations of authigenic pyrite from a Pacific deep sea core, Deep Sea Res., v. 19, pp. 133-138.

Hoffert, M., Karpoff, A. M., Clauer, N., Schaaf, A., Courtois, C., and Pautot, G., 1978. Néoformations et altérations dans trois faciès volcanosédimentaires du Pacifique Sud, Oceanol. Acta, v. 1, pp. 187-202.

Kukal, Z., 1971. Geology of Recent Sediments: London (Academic Press, Inc.), p. 490.

Landergreen, S. and Carjaval, M. C., 1969. Contribution to the geochemistry of boron. III: The relationship between boron concentration in marine clay sediments and the salinity of depositional environment expressed as adsorption isotherm, Arkiv. Mineral. Geol., v. 5, pp. 11-22.

Le Lann, F., 1972. Les phosphorites sous-marines, Rapport B. R. G. M., 72SGN 267MAR, p. 109.

Love, L. G. and Amstutz, G. C., 1966. Review of microscopic pyrite, Fortschr. Miner., v. 43, pp. 273-309.

Marchand, L., 1974. Framboïdes de pyrite in situ, Ann. Soc. Geol. Belgique, v. 97, pp. 561-564.

Morgenstein, M., 1967. Authigenic cementation of scoriaceous deep-sea sediments west of the Society Ridge, South Pacific, Sedimentology, v. 9, pp. 105-118.

Odent, B. and Prouvost, J., 1974. Présence de pyrite framboïdale dans le Kimmeridgien de Boulogne-sur-mer (Pasde-Calais), C. R. Acad. Sci., Paris, t. 279, pp. 1967-1970.

Rikard, D. T., 1970. The origin of framboids, Lithos, v. 3, p. 269-293.

Sakai, H., 1971. Sulfur and oxygen isotopic study of barite concretions from banks in the Japan sea off the Northeast Honshu, Japan, Geochim. J., v. 5, pp. 79-93.

Sheppard, A. R. and Gude, A. J., 1973. Zeolites and associated authigenic silicate minerals in tuffaceous rocks of the Big Sandy Formation, Mohave County, Arizona, Geol. Surv. Prof. Paper, v. 830, p. 36.

Siesser, W. G. and Rogers, J., 1976. Authigenic pyrite and gypsum in South West African continental slope sediments, Sedimentology, v. 23, pp. 567-577.

Staples, L. W., 1965. Zeolite filling and replacement in fossils, Amer. Miner., v. 50, pp. 1796-1801.

Sweeney, R. E. and Kaplan, I. R., 1973. Pyrite framboïd formation: laboratory synthesis and marine sediments, Econ. Geol., v. 68, pp. 618-634.

Tooms, J. S., Summerhayes, C. P., and Cronan, D. S., 1969. Geochemistry of marine phosphorites and manganese deposits, Oceanogr. Mar. Biol. Ann. Rev., v. 7, pp. 49-100.

Turekian, K. K., 1964. The marine geochemistry of strontium, Geochim. Cosmochim. Acta, v. 28, pp. 1479-1496. 1968. Deep sea deposition of barium, cobalt and silver, Geochim. Cosmochim. Acta, v. 32, pp. 603-612. 


\section{PLATE 1}
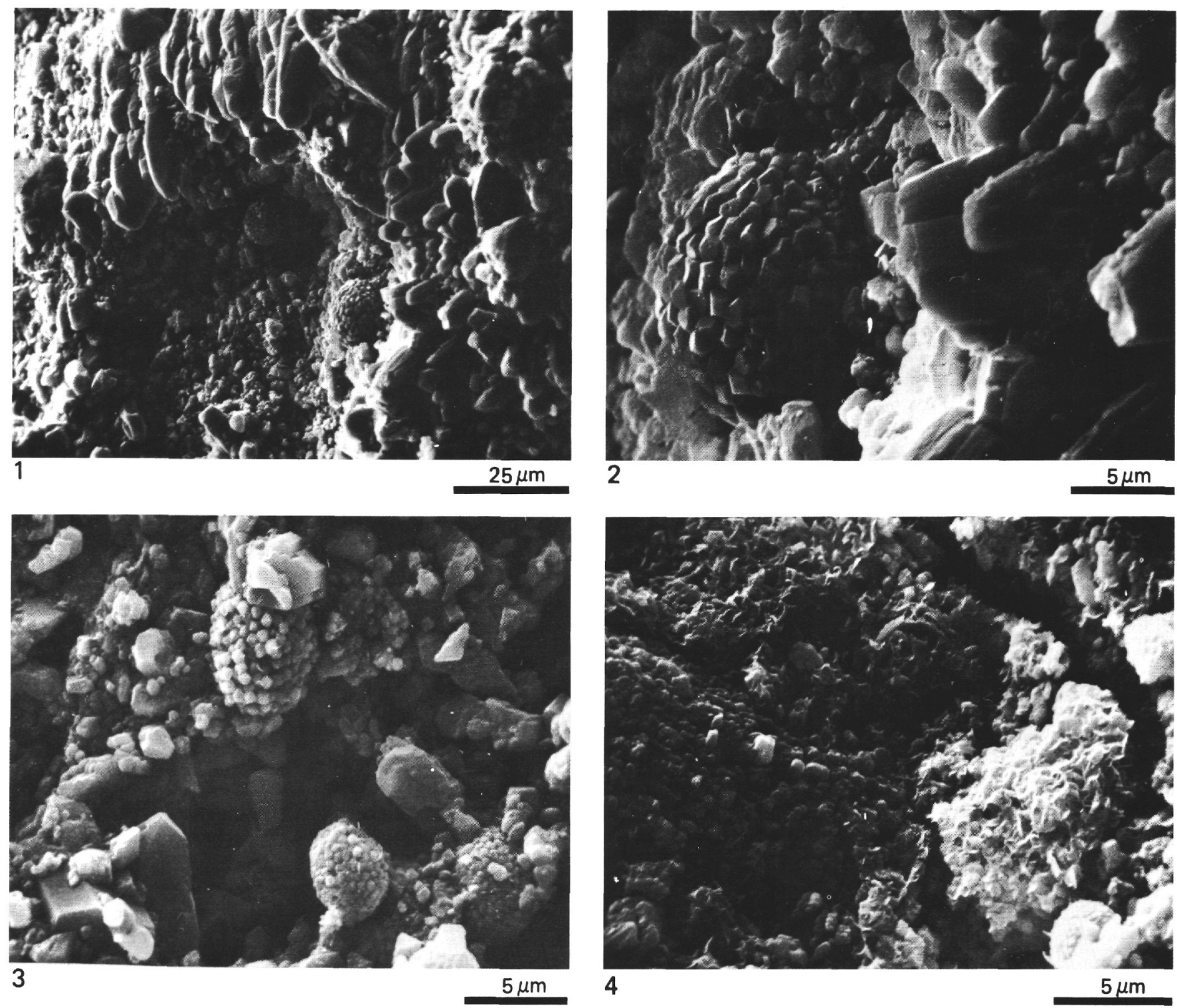

PLATE 1

S.E.M. photomicrographs of bryozoan fragments from reef sand composing Unit 5 at Site 433. Authigenic clays and framboïdal pyrite occur in bryozoan pores which are calcite-recrystallized.

Figure 1 General view of a bryozoan pore with framboïds of pyrite; recrystallized calcite fringes the pore.

Figure 2 High magnification of figure 1, showing framboïd of pyrite.

Figure 3 Very small framboïds of pyrite in another pore.

Figure 4 Authigenic clays as smectites growing in a bryozoan pore. 


\section{PLATE 2}

S. E. M. photomicrographs of particles from the coarse fraction $(>63 \mu \mathrm{m})$ of the volcanic sand making up sedimentary Unit 4 from Hole 433A. (All minerals were determined by X-ray dispersive energy spectroscopy tests.)

Figure 1 Well-preserved crystal of plagioclase. The beginning of alteration is shown by small sheets released from the crystal surface.

Figure 2 In the coarse fraction, well-preserved crystals of plagioclase associated with authigenic twin crystals of zeolites (harmotome).

Figure 3 Authigenic twin crystals of zeolite with a fine deposit of calcareous debris and clays.

Figure 4 The primary twin crystals of zeolite are coated with calcareous debris clays and secondary silicates as zeolites.

Figure $5 \quad$ High magnification of figure 3, showing coating of clays, coccolith fragments, and secondary zeolites.

Figure 6 In the coated material, the primary twin zeolites, small crystals of barite (B) occur with the clays and coccolith fragments.

Figure $7 \quad$ A secondary well-crystallized zeolite growing in pore of cemented primary twin zeolites.

Figure $8 \quad$ Detail of small secondary zeolite (phillipsite or harmotome) with fine deposits of clays.

Figure 9 Finally, the primary twin crystals of zeolite are completely coated and cemented by the secondary deposits (clays, coccoliths, barite, and secondary zeolites) and form rounded aggregated particles. 
PLATE 2

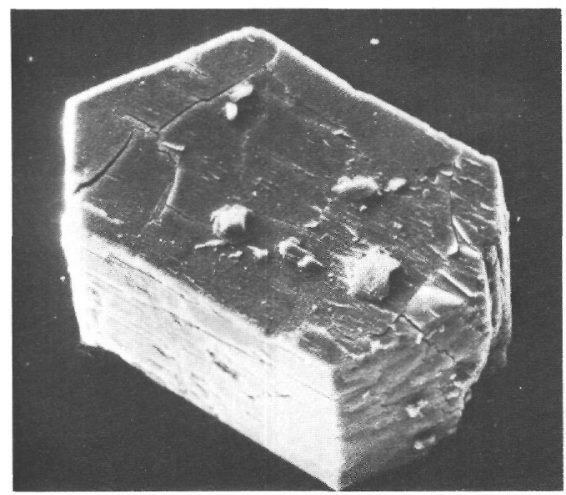

1

$50 \mu \mathrm{m}$

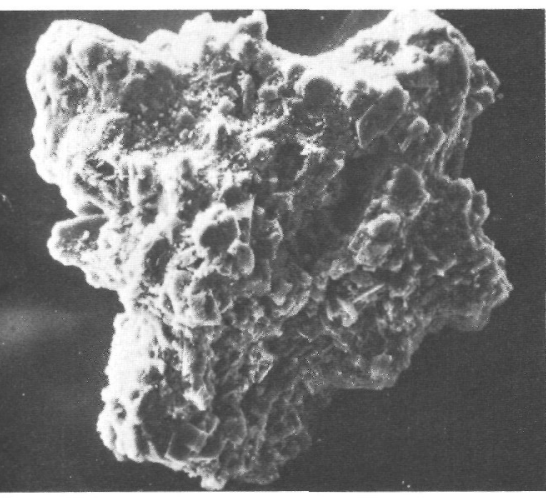

4

$50 \mu \mathrm{m}$
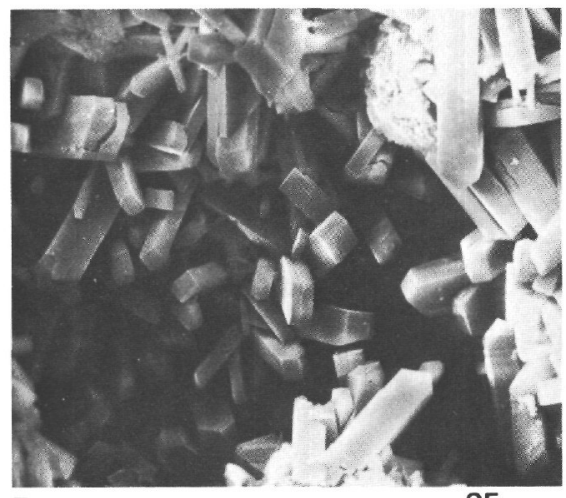

7

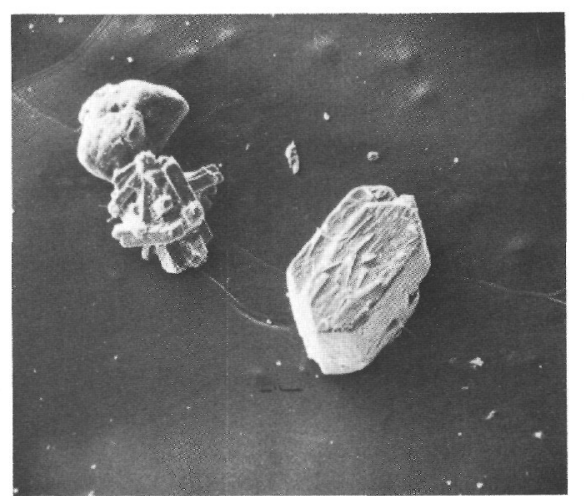

2

$250 \mu \mathrm{m}$

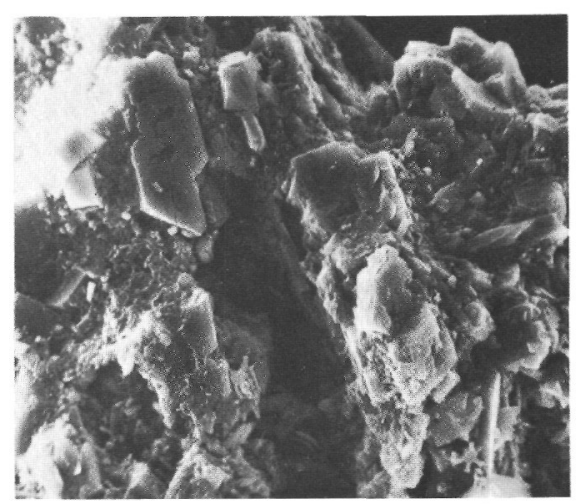

$25 \mu \mathrm{m}$

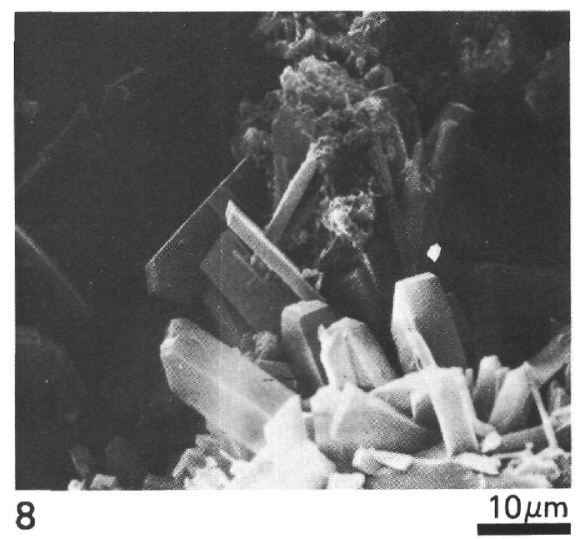

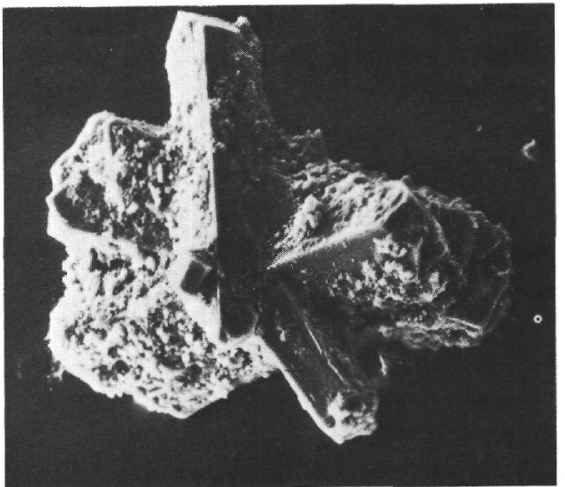
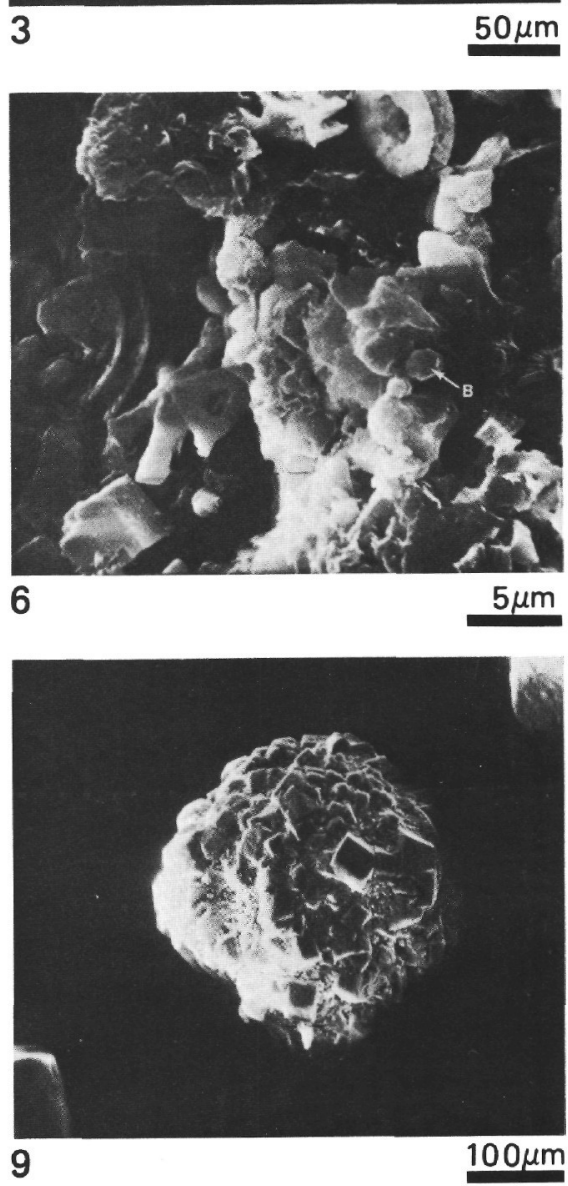